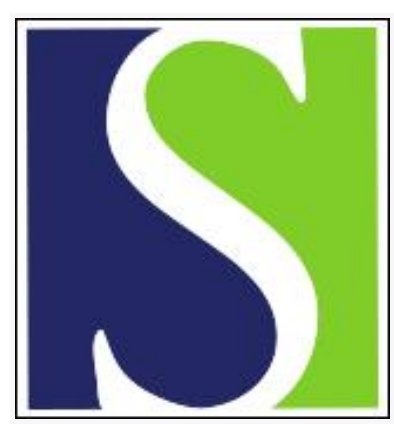

Scand J Work Environ Health Online-first -article

https://doi.org/10.5271/sjweh.2907

Published online: 19 Feb 2010

Exposure to mercury among Norwegian dentists and dental healthcare personnel

by Guzzi G, Pigatto PD, Ronchi A, Minoia C

Affiliation: Italian Association for Metals and Biocompatibility Research (AIRMEB), 20122 Milan, Italy. gianpaolo_guzzi@fastwebnet.it

Refers to the following text of the Journal: $2010 ; 36(3): 231-241$

Key terms: dental healthcare; dental healthcare personnel; dentist; exposure; healthcare personnel; letter to the editor; mercury; Norway

This article in PubMed: www.ncbi.nlm.nih.gov/pubmed/20169292

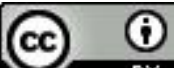




\section{Exposure to mercury among Norwegian dentists and dental healthcare personnel}

Svendsen et al (1) provided an excellent historical overview of chronic exposure to mercury vapors among Norwegian dental workers and found that the systemic level of exposure to mercury is greater among dental nurses than dentists at the workplace. While agreeing with much of what Svendsen et al (1) reported in their investigation, we would like to raise two points.

First, in discussing the value of urine as an indicator of mercury exposure, the authors state: "The measured level of urinary mercury only reflects the exposure level for a relatively short period of the working life. Still, this is the only objective measure on exposure that exists, and we think that a measured urine level may give an indication on the working conditions in the clinic at the time of the measurement." (1) However, there is substantial evidence from studies among humans supporting the view that urinary mercury may not reflect long-term exposure to vapors of mercury generated from the handling of mercury amalgam in dental offices (2). Therefore, mercury in urine is considered a rough biological indicator for long-term exposure to mercury vapor from restorations of mercury-containing amalgam (2-7). Even in acute mercury intoxication, in some cases the determination of mercury concentrations in urine specimens may be of little clinical relevance as a biological medium of the severity of clinical course and symptoms following over-exposure to metallic mercury $(8,6)$.

Second, the authors did not address the current primary source of exposure to mercury vapor for patients and dental staff, namely, the removal of amalgam fillings containing mercury (9-11). During the cutting of dental amalgam filling with a high-speed air turbine, the air concentration of mercury vapor present in the breathing zone may exceed the short-term threshold limit (STEL) for mercury of $0.15 \mathrm{mg} / \mathrm{m}^{3}(10,12)$. After the use of copper amalgam was banned - where liquid metallic mercury was mixed with metals using a mortar and pestle - because of its toxic effects for both dental patients and dental team members and high risk of contaminating the dental office, amalgam-replacement has become the major source of exposure to mercury for dental office staff, mainly in the form of mercury vapors. The conventional approach to removing mercury amalgam is to drill out the entire mercury-based filling, resulting in a significant elevation of mercury vapor in the air of the breathing zone (observed range of elemental mercury vapor was $\left.0.5-1 \mathrm{mg} / \mathrm{m}^{3}\right)(9-12)$.
When dental professionals inhale mercury vapor, about $80 \%$ is believed to be retained in the target tissues (ie, kidneys, brain, endocrine organs, and other tissues). Consequently, the long-term body burden of mercury in dental professionals may be greater than those non-occupationally exposed to mercury vapor (13). As confirmation, in an animal study where dental amalgam dust containing mercury was ground close to the nose of rats, Cutright et al (14) have shown a positive correlation between the inhaled dust and the mercury disposition in the whole blood and tissue of the rats.

By studying the frequency of symptoms among Swedish dental personnel with long-term exposure to mercury amalgam, Nilsson \& Nilsson (2) found that individuals with low urinary mercury showed more symptoms (ie, tremor, short-term memory deficits, fatigue) as compared to those with higher urine mercury levels. The apparent low mercury levels in urine might simply reflect the dental workers' poor renal function that failed to remove the metal from the renal parenchyma due to accumulation and retention of mercury. As always, during pregnancy, female dental healthcare professionals may be exposed to unsafe levels of mercury (12), which are known to damage the developing fetal brain (4).

In their concluding paragraph, Svendsen et al correctly highlight that exposure to mercury in the dental setting varies substantially among dental healthcare workers; we would like to emphasize that the safety of dental workers may be enhanced by increasing awareness of proper mercury hygiene.

\section{References}

1. Svendsen K, Syversen T, Melø I, Hilt B. Historical exposure to mercury among Norwegian dental personnel. Scand J Work Environ Health. 2010;36(3):231-241.

2. Nilsson B, Nilsson B. Mercury in dental practice. II. Urinary mercury excretion in dental personnel. Swed Dent J. 1986;10:221-32.

3. Lorscheider FL, Vimy MJ, Summers AO. Mercury exposure from "silver" tooth fillings: emerging evidence questions a traditional dental paradigm. FASEB J. 1995;9:504-8.

4. Clarkson TW, Magos L. The toxicology of mercury and its chemical compounds. Crit Rev Toxicol. 2006;36:609-62. 
5. Guzzi G, Pigatto PD. Urinary mercury levels in children with amalgam fillings. Environ Health Perspect. 2008;116:286-7.

6. Nuttal KL. Interpreting mercury in blood and urine of individual patients. Ann Clin Lab Sci. 2004;34:235-50.

7. Mutter J, Naumann J, Guethlin C. Comments on the article "The toxicology of mercury and its chemical compounds" by Clarkson and Magos (2006). Crit Rev Tox. 2007;37:537-49.

8. Gattineni J, Weiser S, Becker AM, Baum M. Mercury intoxication: lack of correlation between symptoms and levels. Clin Pediatr. 2007;46:844-6.

9. Cooley RL, Barkmeier WW. Mercury vapor emitted during ultraspeed cutting of amalgam. J Indiana Dent Assoc. 1978;57:28-31.

10. Richards JM, Warren PJ. Mercury vapour released during the removal of old amalgam restorations. Br Dent J. 1985;159:231-2.

11. Guzzi G, Minoia C, Pigatto P, Ronchi A, Gatti A, S. Angeleri, et al. Safe dental amalgam removal in patients with immuno-toxic reactions to mercury. Toxicol Lett. 2003;144 suppl 1:35.

12. Guzzi G, Pigatto PD. Occupational exposure to mercury from amalgams during pregnancy. Occup Environ Med. 2007;64:715-6.
13. Nylander M. Mercury in pituitary glands of dentists. Lancet. 1986;22,1:442.

14. Cutright DE, Miller RA, Battistone GC, Milikan LJ. Systemic mercury levels caused by inhaling mist during high-speed amalgam grinding. J Oral Med. 1973;28:100-4.

Gianpaolo Guzzi, ${ }^{1}$ Paolo D Pigatto, ${ }^{2}$ Anna Ronchi, ${ }^{3}$ Claudio Minoia $^{3}$

1 Italian Association for Metals and Biocompatibility Research (AIRMEB), Milan, Italy.

2 Department of Technology for Health, Dermatological Clinic, IRCCS Galeazzi Hospital, University of Milan, Milan, Italy.

3 Laboratory of Environmental and Toxicology Testing "S. Maugeri" (IRCCS), Pavia, Italy.

Correspondence to: Dr G Guzzi, Italian Association for Metals and Biocompatibility Research (AIRMEB); Via A Banfi, 4; 20122 Milan; Italy. [E-mail: gianpaolo_guzzi@fastwebnet.it] 